\title{
An Unorthodox Approach to Realize the Correlation Between Dielectric Constant and Electronic Disorder in $\mathrm{Cr}-\mathrm{Doped} \mathrm{PrFeO}_{3}$
}

\author{
Anil Kumar*1, Omkar V. Rambadey ${ }^{1}$ and P. R. Sagdeo ${ }^{1 *}$ \\ ${ }^{I}$ Material Research Laboratory, Discipline of Physics and MEMS, Indian Institute of \\ Technology Indore, Indore-453552, India.
}

*Corresponding author email address: prs@iiti.ac.in, garry.anil1369@gmail.com 
(a) Structural characterizations:

The structural phase purity of the prepared samples has been checked through synchrotron x-ray diffraction (SXRD) at BL-12 using Indus-2 synchrotron source[1]. The detailed analysis of SXRD spectra followed by the Rietveld refinements for the prepared series of samples is given in figure-S1. The x-ray diffraction data is best fitted by centrosymmetric Pnma space group (see figure-S1 b) and one to one fit of experimental data with the fitted data confirms the structural phase purity of the samples.
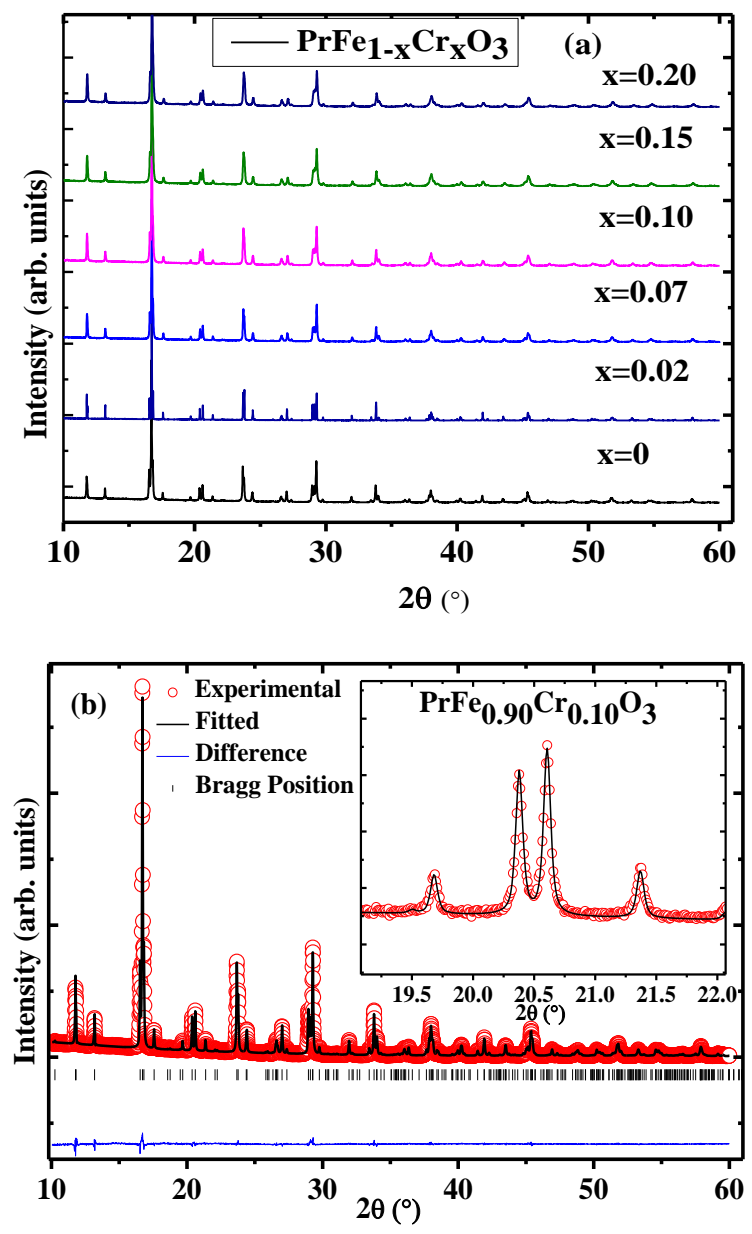

Figure-S1: The x-ray diffraction for prepared series of samples (a), Figure-(b) represents the representative Rietveld refined x-ray diffraction data by considering the Pnma. Inset in the figure-b represents the quality of fitting. 


\section{(b) Raman spectral analysis:}

The vibrational properties of prepared samples has been studied by using LABRAM HR dispersive spectrometer equipped with $633 \mathrm{~nm}$ excitation lasers source having a CCD detector in backscattered mode [2].

Raman spectrum for $\mathrm{Cr}$ doped $\mathrm{PrFeO}_{3}$ samples are illustrated in figure-S2(a). The appearance of new Raman mode around $510 \mathrm{~cm}^{-1}$ and systematic correlation with structural disorder is well understood[2]. In addition to this, origin of Raman mode around $620 \mathrm{~cm}^{-1}$ in $\mathrm{PrFeO}_{3}$ is related to symmetric stretching vibrations of octahedra and is highly sensitive to oxygen stoichiometry[3]. The presence of highly interacted half-filled $e_{g}$ electrons could be responsible for the generation of this mode. At this point, it should be noted here that with the inclusion of $\mathrm{Cr}$ doping, there is a systematic decrease in the intensity of the said breathing mode (as indicated by arrow in fig-S2(a)) which reveals the reduction in oxygen defect density with $\mathrm{Cr}$ doping.

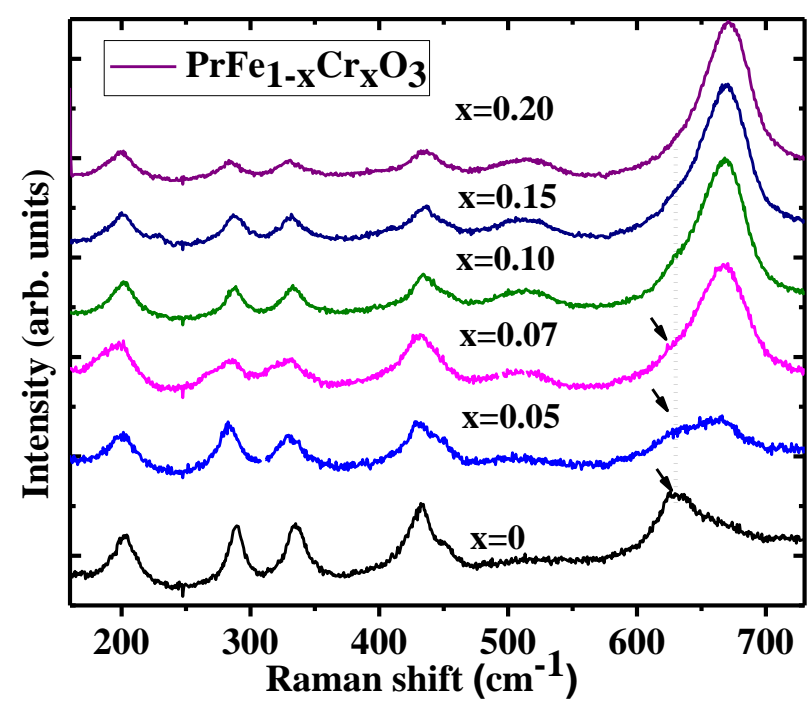

Figure-S2 (a): Raman spectrum for prepared series of samples. Arrow represents the reduction of defects with inclusion of $\mathrm{Cr}$ doping. 


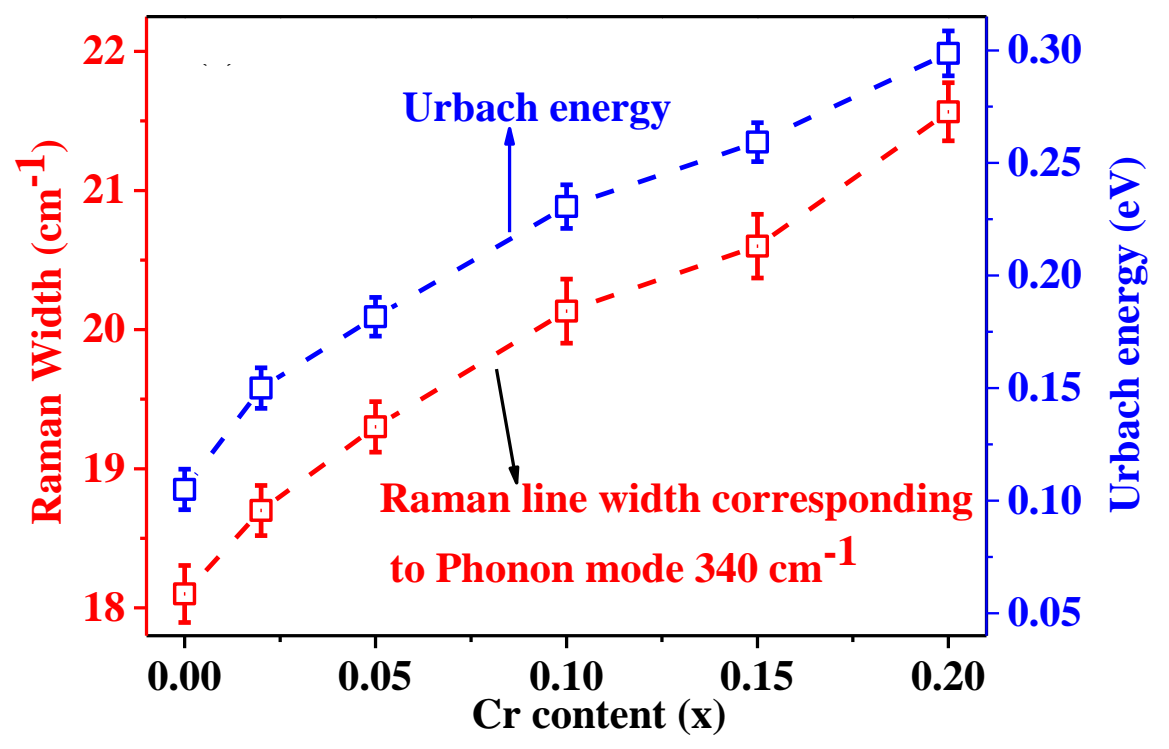

Figure-S2 (b): The systematic variation of Urbach energy and Raman line width as a function of Cr content[1].

In addition to this, symmetric broadening in Raman line shapes has been observed with $\mathrm{Cr}$ doping (depicted in figure-S2(b) in supporting information), which clearly indicates the escalation in compressive strain (structural disorder) with the inclusion of $\mathrm{Cr}$ doping. Hence, it can be concluded that the systematic increase in the values of Urbach energy shows excellent consistency with the disorder estimated from the Raman spectroscopy.

References:

[1] A. Kumar, V. Mishra, M.K. Warshi, A. Sati, A. Sagdeo, R. Kumar, P.R. Sagdeo, Strain induced disordered phonon modes in $\mathrm{Cr}$ doped PrFeO3, Journal of Physics: Condensed Matter. 31 (2019) 275602. https://doi.org/10.1088/1361-648x/ab1195.

[2] A. Kumar, V. Mishra, M. Warshi, A. Sati, A. Sagdeo, R. Kumar, P. R Sagdeo, Strain Induced Disordered Phonon Modes in Cr doped PrFeO3, 2019. https://doi.org/10.1088/1361$648 X / a b 1195$. 
[3] A. Kumar, S. Umrao, P.R. Sagdeo, Orbital facilitated charge transfer originated phonon mode in Cr-substituted PrFeO3: A brief Raman study, Journal of Raman Spectroscopy. (2020). https://doi.org/10.1002/jrs.5894. 